\title{
PROPERTIES OF TRAJECTORIES OF A MULTIFRACTIONAL ROSENBLATT PROCESS
}

UDC 519.21

\author{
GEORGII SHEVCHENKO
}

\begin{abstract}
A Rosenblatt process and its multifractional counterpart are considered. For a multifractional Rosenblatt process, we investigate the local properties of its trajectories, namely the continuity and localizability. We prove the existence of square integrable local times for both processes.
\end{abstract}

\section{INTRODUCTION}

Stochastic processes with long memory (in other words, with long range dependence) remain an extensively developing topic over more than a half of a century because of their numerous applications in modelling various natural phenomena, transferring information in computer nets, dynamics of prices of financial assets, etc.

The long range dependence phenomena is modelled most often with the help of a fractional Brownian motion. In the monograph by Mishura [5], a detailed survey of the literature devoted to the fractional Brownian motion as well as main results of this topic are given. A disadvantage of a fractional Brownian motion that restricts an area of its possible applications is that this process has light tails of the normal distribution. There is a number of studies devoted to processes with long range dependence that have heavier tails. These are, in particular, stable processes (see the book by Samorodnitsky and Taqqu [9]).

An interesting class of processes with long range dependence that have "moderate" tails ("moderate" means that the tails are heavier than those of the normal distribution but lighter than those of a stable distribution), known as Hermite processes, appears in the so-called noncentral limit theorem for strongly dependent random variables proved in the papers by Dobrushin and Major [3] and Taqqu [10,11. The most studied among these processes is the Hermite process of rank 2 defined in the paper by Rosenblatt [8] (the latter is also known as the Rosenblatt process). Among publications devoted to the Rosenblatt process, we mention papers by Pipiras [7], where a wavelet expansion is constructed for this process; Tudor [13], where stochastic analysis with respect to the Rosenblatt process is developed; Albin [1, where the distribution of the maximum of this process is found; and Tudor and Torres [12, where an application of the Rosenblatt process in finance mathematics is considered (namely, the Rosenblatt process is considered in [12] as a model of price evolutions).

2010 Mathematics Subject Classification. Primary 60G22; Secondary 60J55, 60B10.

Key words and phrases. Rosenblatt process, multiple stochastic integral, local time, localizability, fractional Brownian motion.

The author is grateful to the European Commission for a support in the framework of the program "Marie Curie Actions", Grant \# PIRSES-GA-2008-230804. 
It is often the case that local properties of processes vary with time. This suggests an idea that another model is needed to describe such phenomena (recall that all the processes mentioned above, e.g. the fractional Brownian motion, fractional stable processes, and Hermite processes, have stationary increments). A demand in a new model is even higher if the microscopic behavior of a process differs essentially from its macroscopic behavior (this phenomenon often occurs in financial markets where annual fluctuations are much smoother than those observed during a trading session). The latter phenomenon indicates the lack of self-similarity, which is an intrinsic property of the processes mentioned above. This explains why the so-called multifractional processes become popular as models for such cases.

A multifractional Rosenblatt process is defined and some of its properties are studied in this paper. The paper is organized as follows. Section 1 discusses briefly the necessary properties of multiple integrals and local times. A multifractional Rosenblatt process is defined and its basic properties are studied in Section 2. Among those properties are the continuity and localizability. The main results of this paper concerning the existence and quadratic integrability of the local time for the Rosenblatt process and for the multifractional Rosenblatt process are proved in Section 3 .

\section{Preliminaries}

1.1. Multiple integrals. We briefly discuss necessary notions for two-dimensional integrals considered with respect to a Wiener process. More details concerning this topic can be found in 6 .

Let $W$ be a standard Wiener measure on the real line, that is, a random measure with independent values at disjoint sets and such that if $A$ is a set of a finite Lebesgue measure, then $W(A)$ has the normal $N(0, \lambda(A))$ distribution.

The construction of the integral with respect to the measure $W$ is well known for functions $a \in L^{2}(\mathbb{R})$; namely, the integral

$$
I_{1}(a)=\int_{\mathbb{R}} a(x) W(d x)
$$

is defined as the $L^{2}(\Omega)$ limit of integrals of simple functions that approximate $a$ in the space $L^{2}(\mathbb{R})$. The integral defined in this way is a linear isometry acting from $L^{2}(\mathbb{R})$ to $L^{2}(\Omega)$.

The two-dimensional integral is defined similarly. Let $\widehat{L}^{2}\left(\mathbb{R}^{2}\right)$ be the space of square integrable symmetric functions defined on $\mathbb{R}^{2}$; the space $\widehat{L}^{2}\left(\mathbb{R}^{2}\right)$ inherits the Hilbert structure from $L^{2}\left(\mathbb{R}^{2}\right)$. The norm in this space is denoted by $\|\cdot\|$. Let $S$ be the set of simple functions $f \in \widehat{L}^{2}\left(\mathbb{R}^{2}\right)$ of the following form:

$$
f(x, y)=\sum_{k, j=1}^{n} a_{k j} \mathbb{1}_{A_{k}}(x) \mathbb{1}_{A_{j}}(y),
$$

where $A_{1}, \ldots, A_{n}$ are disjoint subsets of $\mathbb{R}$ whose measures are finite, $a_{k j}=a_{j k}$ for all $k, j=1, \ldots, n$ and $a_{k k}=0$ for all $k=1, \ldots, n$. The integral of such a function is defined in the natural way, namely

$$
I_{2}(f)=\iint_{\mathbb{R}^{2}} f(x, y) W(d x) W(d y)=\sum_{k, j=1}^{n} a_{k j} W\left(A_{k}\right) W\left(A_{j}\right) .
$$

It is worthwhile mentioning that the diagonal is excluded from the domain of integration (this corresponds to the assumption that $a_{k k}=0$ ). This is a very important feature of this construction of the integral. The mapping $\frac{1}{2} I_{2}: S \rightarrow L^{2}(\Omega)$ is a linear isometry and thus it is uniquely extended to the closure of the set $S$, that is, to the whole space $\widehat{L}^{2}\left(\mathbb{R}^{2}\right)$. 
In addition to the properties of linearity and isometry (which, in particular, means that $I_{2}$ preserves the Hilbert structure), the mapping $I_{2}$ has some other important properties. We mention three of them below.

1. If $a, b \in L^{2}(\mathbb{R})$ are orthogonal functions and if $f=a \widehat{\otimes} b \in \widehat{L}^{2}\left(\mathbb{R}^{2}\right)$ is the symmetric tensor product, that is, $f(x, y)=(a(x) b(y)+a(y) b(x)) / 2$, then

$$
I_{2}(h)=I_{1}(a) I_{1}(b) .
$$

2. For all $a \in L^{2}(\mathbb{R})$, we have $I_{2}(a \widehat{\otimes} a)=\left(I_{1}(a)\right)^{2}-\|a\|_{L^{2}(\mathbb{R})}^{2}$.

3. The preceding property implies the following important result concerning the distribution of $I_{2}(f)$. A function $f \in \widehat{L}^{2}\left(\mathbb{R}^{2}\right)$ can be identified with a self-adjoint operator $M_{f}$ on $L^{2}(\mathbb{R})$ defined by

$$
\left(M_{f} a\right)(x)=\int_{\mathbb{R}} f(x, y) a(y) d y .
$$

Let $\lambda_{k, f}, k \geq 1$, be the eigenvalues of this operator (the total number of the eigenvalues can be finite, countable, or even uncountable) ordered with respect to the values of their modulus. Let $\varphi_{k, f}, k \geq 1$, be the corresponding orthonormal eigenfunctions. As is well known,

$$
f(x, y)=\sum_{k \geq 1} \lambda_{k, f} \varphi_{k, f} \widehat{\otimes} \varphi_{k, f},
$$

whence, by the preceding property,

$$
I_{2}(f)=\sum_{k \geq 1} \lambda_{k, f}\left(\zeta_{k}^{2}-1\right),
$$

where the random variables $\zeta_{k}=I_{1}\left(\varphi_{k, f}\right)$ are independent and have the standard normal distribution. Therefore $I_{2}(f)$ has a generalized chi-square distribution. Thus the characteristic function of $I_{2}(f)$ is equal to

$$
\mathrm{E}\left[e^{i \alpha I_{2}(f)}\right]=\prod_{k \geq 1} \mathrm{E}\left[e^{i \alpha \lambda_{k, f}\left(\zeta_{k}^{2}-1\right)}\right]=\prod_{k \geq 1} \frac{e^{-i \alpha \lambda_{k, f}}}{\sqrt{1-2 i \alpha \lambda_{k, f}}} .
$$

Since $\sum_{k \geq 1} \lambda_{k, f}^{2}=\|f\|^{2}<\infty$, the latter product converges for all $\alpha \in \mathbb{R}$. The characteristic function can be rewritten in terms of the cumulants, namely

$$
\ln \mathrm{E}\left[e^{i \alpha I_{2}(f)}\right]=\sum_{n \geq 2} \kappa_{n} \frac{(i \alpha)^{n}}{n !}
$$

where

$$
\begin{aligned}
\kappa_{n}=2^{n-1}(n-1) ! \sum_{k \geq 1} \lambda_{k, f}^{n}=2^{n-1}(n-1) ! \operatorname{tr} M_{f}^{n} & \\
=2^{n-1}(n-1) ! \int \cdots \int_{\mathbb{R}^{n}} f\left(x_{1}, x_{2}\right) & f\left(x_{2}, x_{3}\right) \cdots \\
& \quad \times f\left(x_{n-1}, x_{n}\right) f\left(x_{n}, x_{1}\right) d x_{1} d x_{2} \cdots d x_{n} .
\end{aligned}
$$

We obtain from relation (2) that

$$
\begin{aligned}
\left|\mathrm{E}\left[e^{i \alpha I_{2}(f)}\right]\right| & =\left(\prod_{m \geq 1}\left(1+4 \alpha^{2} \lambda_{k, f}^{2}\right)\right)^{-1 / 4} \\
& \leq\left(1+4 \alpha^{2} \sum_{k \geq 1} \lambda_{k, f}^{2}+16 \alpha^{4} \sum_{j<k} \lambda_{j, f}^{2} \lambda_{k, f}^{2}+64 \alpha^{6} \sum_{j<k<l} \lambda_{j, f}^{2} \lambda_{k, f}^{2} \lambda_{l, f}^{2}\right)^{-1 / 4} .
\end{aligned}
$$


1.2. Local times. Let $\left\{Z_{t}, t \geq 0\right\}$ be a separable stochastic process. The sojourn measure for $Z$ is defined as follows:

$$
\mu(A, B)=\lambda\left(\left\{s \in A, Z_{s} \in B\right\}\right),
$$

where $A \in \mathcal{B}([0, \infty))$ and $B \in \mathcal{B}(\mathbb{R})$. If $\mu(A, \cdot)$ is absolutely continuous with respect to the Lebesgue measure on $\mathbb{R}$, then we say that $Z$ has the local time in the set $A$; the local time is defined as the Radon-Nikodym derivative of the function $\mu(A, \cdot)$ :

$$
L(A, x)=\frac{d \mu(A, \cdot)}{d \lambda}(x) .
$$

We also use the notation $L(t, x)=L([0, t], x)$ when it is not misleading.

The analytic method due to Berman [2] can briefly be described as follows. For a fixed trajectory of a process, the Fourier transform

$$
F(u)=\int_{\mathbb{R}} e^{i u x} L(t, x) d x
$$

of $L(t, x)$ with respect to the variable $x$ can be represented with the help of the local time as follows:

$$
F(v)=\int_{0}^{t} e^{i v Z_{s}} d s
$$

Then the local time can be represented via the inverse Fourier transform of this function, that is,

$$
L(t, x)=\frac{1}{2 \pi} \int_{\mathbb{R}} \int_{0}^{t} e^{i v\left(Z_{s}-x\right)} d s d v .
$$

The following criterion for the existence of the square integrable local time is a corollary of the latter equality.

Proposition 1.1 ([2]). A stochastic process $\left\{Z_{t}, t \geq 0\right\}$ has the local time

$$
L(A, x) \in L^{2}(\mathbb{R} \times \Omega)
$$

if and only if

$$
K=\int_{\mathbb{R}} \int_{A} \int_{A} \mathrm{E}\left[e^{i v\left(Z_{t}-Z_{s}\right)}\right] d s d t d v<\infty
$$

In this case,

$$
\int_{\mathbb{R}} \mathrm{E}\left[L^{2}(A, x)\right] d x=K
$$

\section{Definitions}

The Rosenblatt process arises as the limit of normalized sums of strongly dependent random variables (see [3]).

Definition 2.1. Let $H \in(1 / 2,1)$. Then

$$
Z_{t}^{H}=c(H) \iint_{\mathbb{R}^{2}} \int_{0}^{t}(s-x)_{+}^{H / 2-1}(s-y)_{+}^{H / 2-1} d s W(d x) W(d y)
$$

is called the Rosenblatt process with the Hurst parameter $H$, where

$$
c(H)=\frac{\sqrt{2 H(2 H-1)}}{\mathrm{B}(H / 2,1-H)}
$$

and where B is the Euler beta function. 
It is known (see, for example, [13]) that the Rosenblatt process has the following properties:

(1) self-similarity: $Z^{H}$ is $H$-self-similar; that is, the processes

$$
\left\{Z^{H}(c t), t \geq 0\right\} \quad \text { and } \quad\left\{c^{H} Z^{H}(t), t \geq 0\right\}
$$

have identical finite-dimensional distributions for all $c>0$;

(2) stationary increments: $Z^{H}$ has stationary increments; that is, the finitedimensional distributions of the process $\left\{Z^{H}(t+s)-Z^{H}(s), t \geq 0\right\}$ do not depend on $s \geq 0$;

(3) existence of moments: all the moments of the process $Z^{H}$ are finite; its covariance function coincides with the covariance function of a standard fractional Brownian motion with the Hurst parameter $H$ :

$$
\mathrm{E}\left[Z_{t}^{H} Z_{s}^{H}\right]=\frac{1}{2}\left(t^{2 H}+s^{2 H}-|t-s|^{2 H}\right) ;
$$

(4) continuity: the trajectories of the Rosenblatt process are Hölder continuous of an arbitrary order $\delta<H$.

The multifractional analogue of the Rosenblatt process is defined in a natural way. Let a continuous function $H:[0, \infty) \rightarrow(1 / 2,1)$ be given (it will play the role of the Hurst parameter). For simplicity, we do not normalize the process as in the preceding definition.

Definition 2.2. The multifractional Rosenblatt process with the Hurst function $H$ (or, with a functional parameter $H$ ) is given by

$$
X_{t}=\iint_{\mathbb{R}^{2}} \int_{0}^{t}(s-x)_{+}^{H(t) / 2-1}(s-y)_{+}^{H(t) / 2-1} d s W(d x) W(d y) .
$$

For the sake of simplicity, we restrict our consideration to a finite interval $[0, T]$ in what follows. Put $\hat{H}=\min _{t \in[0, T]} H(t)$ and $\check{H}=\max _{t \in[0, T]} H(t)$. Since $H$ is continuous and its values belong to the interval $(1 / 2,1)$, we conclude that $1 / 2<\hat{H} \leq \check{H}<1$. We further assume that the function $H$ is Hölder continuous of an order $\gamma>\check{H}$, that is,

$$
|H(t)-H(s)| \leq C|t-s|^{\gamma} \text {. }
$$

In fact, all the results given below are true for a more general case where the latter condition is satisfied locally. The local version of (7) means that, for all $u>0$, there exist two numbers $\varepsilon>0$ and $\gamma>\sup _{t \in(u-\varepsilon, u+\varepsilon)} H(t)$ such that inequality (7) holds for all $t, s \in(u-\varepsilon, u+\varepsilon)$. Nevertheless we assume the global version of this condition in order to make reasoning simpler and statements more transparent.

In what follows, the symbol $C$ denotes a constant whose value may depend on $\hat{H}, \check{H}$, $\gamma, T$, and on the constant involved in (7) only. If a constant depends on other parameters (or if we want to indicate explicitly the dependence of $C$ on certain parameters) we use the notation $C$ with the corresponding subscripts.

For convenience, we denote by $f_{H}(t, x, y)=(t-x)_{+}^{H / 2-1}(t-y)_{+}^{H / 2-1}$ the function involved in the definition of the Rosenblatt process. Consider the following two-parameter random field:

$$
Z(t, H)=\frac{1}{c(H)} Z_{t}^{H}=\iint_{\mathbb{R}^{2}} \int_{0}^{t} f_{H}(s, x, y) d s W(d x) W(d y) .
$$

The following continuity property for $Z(t, H)$ with respect to the parameter $H$ plays an important role in what follows. 
Proposition 2.3. For all $H^{\prime}, H^{\prime \prime} \in[\hat{H}, \check{H}]$,

$$
\mathrm{E}\left[\left(Z\left(t, H^{\prime}\right)-Z\left(t, H^{\prime \prime}\right)\right)^{2}\right] \leq C\left(H^{\prime}-H^{\prime \prime}\right)^{2} .
$$

Proof. We agree that $0 \cdot \ln 0=0$. Then

$$
\begin{aligned}
& \mathrm{E}\left[\left(Z\left(t, H^{\prime}\right)-Z\left(t, H^{\prime \prime}\right)\right)^{2}\right] \\
& \quad=\mathrm{E}\left[\left(\iint_{\mathbb{R}^{2}} \int_{0}^{t}\left(f_{H^{\prime}}(s, x, y)-f_{H^{\prime \prime}}(s, x, y)\right) d s W(d x) W(d y)\right)^{2}\right] \\
& \quad=\iint_{\mathbb{R}^{2}}\left(\int_{0}^{t}\left(f_{H^{\prime}}(s, x, y)-f_{H^{\prime \prime}}(s, x, y)\right) d s\right)^{2} d x d y \\
& \quad=\frac{1}{4} \iint_{\mathbb{R}^{2}}\left(\int_{H^{\prime}}^{H^{\prime \prime}} \int_{0}^{t} f_{h}(s, x, y) \ln \left((s-x)_{+}(s-y)_{+}\right) d s d h\right)^{2} d x d y \\
& \leq C\left|H^{\prime}-H^{\prime \prime}\right| \iint_{\mathbb{R}^{2}} \int_{H^{\prime}}^{H^{\prime \prime}}\left(\int_{0}^{t} f_{h}(s, x, y) \ln \left((s-x)_{+}(s-y)_{+}\right) d s\right)^{2} d h d x d y .
\end{aligned}
$$

We choose a positive $\varepsilon<(\hat{H}-1 / 2) \wedge(1-\check{H})$. Using the inequality $|\ln x| \leq C_{\varepsilon}\left(x^{\varepsilon}+x^{-\varepsilon}\right)$, we obtain

$$
\begin{aligned}
& \mathrm{E}\left[\left(Z\left(t, H^{\prime}\right)-Z\left(t, H^{\prime \prime}\right)\right)^{2}\right] \\
& \quad \leq C_{\varepsilon}\left|H^{\prime}-H^{\prime \prime}\right| \int_{H^{\prime}}^{H^{\prime \prime}} \iint_{\mathbb{R}^{2}}\left(\int_{0}^{t}\left(f_{h-\varepsilon}(s, x, y) d s+f_{h+\varepsilon}(s, x, y)\right) d s\right)^{2} d x d y d h .
\end{aligned}
$$

Now we rewrite the squared expression on the right hand side of the latter inequality as a product of the integrals with respect to different variables (for example, with respect to $s_{1}$ and $s_{2}$ ). As a result, the integrand becomes the sum of four functions

$$
f_{h \pm \varepsilon}\left(s_{1}, x, y\right) f_{h \pm \varepsilon}\left(s_{2}, x, y\right) .
$$

The integrals of these functions are estimated similarly; thus we may restrict the consideration to one of them, to $f_{h+\varepsilon}\left(s_{1}, x, y\right) f_{h+\varepsilon}\left(s_{2}, x, y\right)$, for example. We estimate the integral with respect to $d x$ of the function $f_{h+\varepsilon}\left(s_{1}, x, y\right) f_{h+\varepsilon}\left(s_{2}, x, y\right)$ (more precisely, the integral of its part depending on $x$ ) under the assumption that $s=s_{2}-s_{1}>0$ :

$$
\begin{aligned}
\int_{-\infty}^{s_{1}} & \left(s_{1}-x\right)^{(h+\varepsilon) / 2-1}\left(s_{2}-x\right)^{(h+\varepsilon) / 2-1} d x \\
& =\mid \text { change } x \rightarrow s_{1}-s y \mid \\
& =s^{h+\varepsilon-1} \int_{0}^{\infty} y^{(h+\varepsilon) / 2-1}(1+y)^{(h+\varepsilon) / 2-1} d y \\
& =s^{h+\varepsilon-1} \mathrm{~B}(h+\varepsilon, 1-h-\varepsilon) \leq C_{\varepsilon} s^{h+\varepsilon-1} .
\end{aligned}
$$

The latter estimate is due to the choice of $\varepsilon$. Denoting for a moment

$$
h(s)=|s|^{2(h-\varepsilon)-2}+|s|^{2 h-2}+|s|^{2(h+\varepsilon)-2}
$$

we obtain

$$
\begin{aligned}
& \mathrm{E}\left[\left(Z\left(t, H^{\prime}\right)-Z\left(t, H^{\prime \prime}\right)\right)^{2}\right] \\
& \quad \leq C_{\varepsilon}\left|H^{\prime}-H^{\prime \prime}\right| \int_{H^{\prime}}^{H^{\prime \prime}} \iint_{[0, T]^{2}} h\left(s_{1}-s_{2}\right) d s_{1} d s_{2} d h \\
& \quad \leq C_{T, \varepsilon}\left(H^{\prime}-H^{\prime \prime}\right)^{2} .
\end{aligned}
$$


The latter estimate is valid due to the choice of $\varepsilon$, since

$$
2(h-\varepsilon)-2 \geq 2(\hat{H}-\varepsilon)-2>-1 .
$$

Proposition 2.3 is proved.

Now we estimate the increments of the multifractional Rosenblatt process $X$ defined by equality (6).

Proposition 2.4. If $t, s \in[0, T]$ are such that the distance $|t-s|$ is sufficiently small, then

$$
\mathrm{E}\left[\left(X_{t}-X_{s}\right)^{2}\right] \leq C|t-s|^{2 H(u)}
$$

for all $u \in[t, s]$.

Proof. Since

$$
\begin{aligned}
\mathrm{E}\left[\left(X_{t}-X_{s}\right)^{2}\right]= & \mathrm{E}\left[(Z(t, H(t))-Z(s, H(s)))^{2}\right] \\
\leq & 3\left(\mathrm{E}\left[(Z(t, H(t))-Z(t, H(u)))^{2}\right]+\mathrm{E}\left[(Z(s, H(s))-Z(s, H(u)))^{2}\right]\right. \\
& \left.\quad+\mathrm{E}\left[(Z(t, H(u))-Z(s, H(u)))^{2}\right]\right) \\
\leq & C(H(t)-H(u))^{2}+C(H(s)-H(u))^{2}+(t-s)^{2 H(u)} / c^{2}(H(u)) \\
\leq & C|t-s|^{2 \gamma}+(t-s)^{2 H(u)} / c^{2}(H(u)) \\
\leq & C|t-s|^{2 H(u)}\left(|t-s|^{2 \gamma-2 \breve{H}}+1\right),
\end{aligned}
$$

we complete the proof of Proposition 2.4 in view of $\gamma>\check{H}$.

Theorem 2.5. The trajectories of a multifractional Rosenblatt process are continuous almost surely. Moreover, the trajectories are Hölder continuous on each interval

$$
[a, b] \subset[0, T]
$$

of an arbitrary order $\delta<\min _{t \in[a, b]} H(t)$.

Proof. From Proposition 2.4 and from an estimate for moments of multiple integrals (see [14, Corollary 7.36]) we derive that

$$
\mathrm{E}\left[\left(X_{t}-X_{s}\right)^{2 p}\right] \leq C_{p}|t-s|^{2 \hat{H}(a, b) p}
$$

for an arbitrary natural number $p \geq 1$ and for all $t, s \in[a, b]$, where

$$
\hat{H}(a, b)=\min _{t \in[a, b]} H(t) .
$$

According to the Kolmogorov-Chentsov theorem, the process $X$ is Hölder continuous of an arbitrary order $\delta<2 \hat{H}(a, b) p-1 /(2 p)$. Letting $p \rightarrow \infty$, we complete the proof of Theorem 2.5.

2.1. Localizability. The following definition allows one to describe the behavior of a stochastic process in a neighborhood of a certain point.

Definition 2.6. We say that a process $\left\{Z_{t}, t \geq 0\right\}$ is $\alpha$-localizable at a point $t_{0}$ with a local version $\left\{Y_{t}, t \geq 0\right\}$ if the processes $\left\{\delta^{-\alpha}\left(Z_{t_{0}+\delta t}-Z\left(t_{0}\right)\right), t \geq 0\right\}$ weakly converge (in the sense of the convergence of finite-dimensional distributions) to $\left\{Y_{t}, t \geq 0\right\}$ as $\delta \rightarrow 0+$.

If the weak convergence in this definition is understood as the convergence of distributions in $C[0, A]$ for an arbitrary $A>0$, then we say that the process $Z$ is strongly localizable at the point $t_{0}$. 
Since the limit is unique, the local version, if it exists, is an $\alpha$-self-similar process. For this reason, the property of localizability is also called the property of local self-similarity.

Proposition 2.7. Every multifractional Rosenblatt process (6) is localizable at every point $t_{0} \geq 0$ with the local version $Z\left(\cdot, H\left(t_{0}\right)\right)$.

Proof. Let $Y_{t}^{\delta}=\delta^{-H\left(t_{0}\right)}\left(X_{t_{0}+\delta t}-X\left(t_{0}\right)\right)$. Then

$$
Y_{t}^{\delta}=\delta^{-H\left(t_{0}\right)}\left(Z\left(t_{0}+\delta t, H\left(t_{0}+\delta t\right)\right)-Z\left(t_{0}, H\left(t_{0}\right)\right)\right)=Y_{t}^{\delta, 1}+Y_{t}^{\delta, 1},
$$

where

$$
\begin{aligned}
& Y_{t}^{\delta, 1}=\delta^{-H\left(t_{0}\right)}\left(Z\left(t_{0}+\delta t, H\left(t_{0}\right)\right)-Z\left(t_{0}, H\left(t_{0}\right)\right)\right), \\
& Y_{t}^{\delta, 2}=\delta^{-H\left(t_{0}\right)}\left(Z\left(t_{0}+\delta t, H\left(t_{0}+\delta t\right)\right)-Z\left(t_{0}+\delta t, H\left(t_{0}\right)\right)\right) .
\end{aligned}
$$

Since $\left\{Z\left(t, H\left(t_{0}\right)\right), t \geq 0\right\}$ is a Rosenblatt process multiplied by a nonrandom constant, it has stationary increments and is $H\left(t_{0}\right)$-self-similar. Thus $\left\{Y_{t}^{\delta, 1}, t \geq 0\right\}$ has the same finite-dimensional distributions as the process $\left\{Z\left(t, H\left(t_{0}\right)\right), t \geq 0\right\}$. Hence the random vector $\left(Y_{t_{1}}^{\delta, 1}, \ldots, Y_{t_{1}}^{\delta, 1}\right)$ converges in probability to $\left(Z\left(t_{1}, H\left(t_{0}\right)\right), \ldots, Z\left(t_{n}, H\left(t_{0}\right)\right)\right)$ for all arguments $t_{1}, t_{2}, \ldots, t_{n}$.

Proposition 2.3 implies that

$$
\mathrm{E}\left[\left(Y_{t}^{\delta, 2}\right)^{2}\right] \leq C \delta^{-2 H\left(t_{0}\right)} \delta^{2 \gamma}=C \delta^{2\left(\gamma-H\left(t_{0}\right)\right)} \rightarrow 0, \quad \delta \rightarrow 0 .
$$

Given an arbitrary $t$, we get $Y_{t}^{\delta, 2} \stackrel{\mathrm{P}}{\longrightarrow} 0$ as $\delta \rightarrow 0+$, whence we deduce that the vector $\left(Y_{t_{1}}^{\delta, 2}, \ldots, Y_{t_{1}}^{\delta, 2}\right)$ converges in probability to $(0, \ldots, 0)$ for all $t_{1}, t_{2}, \ldots, t_{n}$.

Applying Slutsky's theorem, we obtain the weak convergence desired.

Theorem 2.8. A multifractional Rosenblatt process is strongly localizable at every point $t_{0} \geq 0$ with the local version $Z\left(\cdot, H\left(t_{0}\right)\right)$.

Proof. We have already established the convergence of finite-dimensional distributions. It remains to check that the family of distributions of the processes

$$
Y_{t}^{\delta}=\delta^{-H\left(t_{0}\right)}\left(Z\left(t_{0}+\delta t, H\left(t_{0}+\delta t\right)\right)-Z\left(t_{0}, H\left(t_{0}\right)\right)\right)
$$

is weakly relatively compact in $C[0, A]$. By Prokhorov's criterion, this property is equivalent to the following two conditions:

(1) the family of random variables $\left\{Y_{0}^{\delta}, \delta>0\right\}$ is bounded in probability;

(2) for all $\varepsilon>0$,

$$
\lim _{\eta \rightarrow 0+} \limsup _{\delta \rightarrow 0+} \mathrm{P}\left\{\sup _{t, s \in[0, A],|t-s|<\eta}\left|Y_{t}^{\delta}-Y_{s}^{\delta}\right| \geq \varepsilon\right\} \rightarrow 0 .
$$

The first condition is obvious in our case.

Further,

$$
\begin{aligned}
P(\delta, \eta, \varepsilon) & =\mathrm{P}\left\{\sup _{|t-s|<\eta}\left|Y_{t}^{\delta}-Y_{s}^{\delta}\right| \geq \varepsilon\right\}=\mathrm{P}\left\{\sup _{|t-s|<\eta}\left|Y_{t}^{\delta}-Y_{s}^{\delta}\right| \geq \varepsilon\right\} \\
& \leq \varepsilon^{-2 p} \mathrm{E}\left[\sup _{|t-s|<\eta}\left(Y_{t}^{\delta}-Y_{s}^{\delta}\right)^{2 p}\right] \leq \varepsilon^{-2 p} \eta^{2 p \rho-1} \mathrm{E}\left[\sup _{|t-s|<\eta} \frac{\left(Y_{t}^{\delta}-Y_{s}^{\delta}\right)^{2 p}}{|t-s|^{2 p \rho-1}}\right] \\
& \leq C_{p, \rho, A} \varepsilon^{-2 p} \eta^{2 p \rho-1} \iint_{[0, A]^{2}} \frac{\mathrm{E}\left[\left(Y_{t}^{\delta}-Y_{s}^{\delta}\right)^{2 p}\right]}{|t-s|^{2 p \rho+1}} d s d t
\end{aligned}
$$


for $p \geq 1$ and $\rho>1 / p$, where the latter bound follows from the Garsia-Rodemich-Ramsey inequality (see [4, Theorem 1.4]). Since

$$
Y_{t}^{\delta}-Y_{s}^{\delta}=\delta^{-H\left(t_{0}\right)}\left(X\left(t_{0}+\delta t\right)-X\left(t_{0}+\delta s\right)\right)
$$

we obtain from Proposition 2.4 and from a bound for moments of multiple stochastic integrals [14, Corollary 7.3.6] that

$$
\begin{aligned}
\mathrm{E}\left[\left(Y_{t}^{\delta}-Y_{s}^{\delta}\right)^{2 p}\right] & \leq C \delta^{-2 H\left(t_{0}\right) p}(\delta|t-s|)^{2 H\left(t_{0}+\delta t\right) p} \\
& =C \delta^{2\left(H\left(t_{0}+\delta t\right)-H\left(t_{0}\right)\right) p}|t-s|^{2 H\left(t_{0}+\delta t\right)} \leq C_{A, p}|t-s|^{2 H\left(t_{0}+\delta t\right) p} \\
& \leq C_{A, p}|t-s|^{2 \hat{H} p}
\end{aligned}
$$

for a sufficiently small $\delta$. Above we have used the property that

$$
\begin{aligned}
\ln \left(\delta^{2\left(H\left(t_{0}+\delta t\right)-H\left(t_{0}\right)\right) p}\right) & \leq 2 p|\ln \delta|\left|H\left(t_{0}+\delta t\right)-H\left(t_{0}\right)\right| \\
& \leq C p A^{\gamma}|\ln \delta| \delta^{\gamma} \rightarrow 0, \quad \delta \rightarrow 0+.
\end{aligned}
$$

Choosing $\rho=\hat{H} / 2$ and $p=2 / \rho$ we obtain

$$
P(\delta, \eta, \varepsilon) \leq C_{A} \varepsilon^{-8 / \hat{H}} \eta \rightarrow 0, \quad \eta \rightarrow 0+,
$$

and this is what was to be proved.

\section{Existence of the local time for a Rosenblatt process and for its MULTIFRACTIONAL ANALOGUE}

In contrast to other results of this paper, the following theorem concerns the usual Rosenblatt process. Theorem 3.1 is interesting in its own, since the existence of the local time has not been investigated so far for the Rosenblatt process. Moreover, one can use it to derive a similar result for a multifractional Rosenblatt process.

Theorem 3.1. A Rosenblatt process has the square integrable local time in each finite interval $[0, T]$.

Proof. By Proposition [1.1, we need to check condition (5) for $A=[0, T]$. Since a Rosenblatt process is $H$-self-similar and has homogeneous increments,

$$
\mathrm{E}\left[e^{i v\left(Z_{t}^{H}-Z_{s}^{H}\right)}\right]=\mathrm{E}\left[e^{i v Z_{|t-s|}^{H}}\right]=\mathrm{E}\left[e^{i v|t-s|^{H} Z_{1}}\right] .
$$

In addition, $Z_{1}=I_{2}(\phi)$, where

$$
\phi(x, y)=\phi_{H}(x, y)=c(H) \int_{0}^{1} f_{H}(s, x, y) d s .
$$

Using bound (44), we get

$$
\mathrm{E}\left[e^{i v|t-s|^{H} Z_{1}}\right]=\mathrm{E}\left[e^{i v|t-s|^{H} I_{2}(\phi)}\right] \leq\left(1+64 v^{6}|t-s|^{6 H} \lambda_{1, \phi}^{2} \lambda_{2, \phi}^{2} \lambda_{3, \phi}^{2}\right)^{-1 / 4} .
$$

Below we prove that $\operatorname{rk} M_{\phi}>2$, whence $\lambda=\lambda_{1, \phi}^{2} \lambda_{2, \phi}^{2} \lambda_{3, \phi}^{2}>0$ (recall that the eigennumbers $\lambda_{k, \phi}$ are ordered according to their absolute values).

Hence

$$
\begin{aligned}
\int_{\mathbb{R}} \int_{0}^{T} \int_{0}^{T} \mathrm{E}\left[e^{i v\left(Z_{t}-Z_{s}\right)}\right] d s d t d v & \leq \int_{0}^{T} \int_{0}^{T} \int_{\mathbb{R}}\left(1+64 \lambda v^{6}|t-s|^{6 H}\right)^{-1 / 4} d v d s d t \\
& =\int_{0}^{T} \int_{0}^{T} \frac{1}{2}|t-s|^{-H} \int_{\mathbb{R}}\left(1+\lambda z^{6}\right)^{-1 / 4} d z d s d t<\infty
\end{aligned}
$$

and this is what was to be proved. 
Proof of the inequality $\operatorname{rk} M_{\phi}>2$. Assume that $\operatorname{rk} M_{\phi} \leq 2$. Let $f_{1}(x)=\mathbb{1}_{[0,1]}(x)$,

$$
f_{2}(x)=\mathbb{1}_{[0,2 / 3]}(x),
$$

and $f_{3}(x)=\mathbb{1}_{[1 / 3,1]}(x)$. Since $\operatorname{rk} M_{\phi} \leq 2$, there exist numbers $\alpha_{1}, \alpha_{2}$, and $\alpha_{3}$, not all being zero, such that $\alpha_{1} M_{\phi} f_{1}+\alpha_{2} M_{\phi} f_{2}+\alpha_{3} M_{\phi} f_{3}=0$. Let $f=\alpha_{1} f_{1}+\alpha_{2} f_{2}+\alpha_{3} f_{3}$. Then

$$
\begin{aligned}
0 & =\left(M_{\phi} f, f\right)=c(H) \iint_{\mathbb{R}^{2}} \int_{0}^{1}(s-x)_{+}^{H / 2-1}(s-y)_{+}^{H / 2-1} f(x) f(y) d s d x d y \\
& =c(H) \int_{0}^{1}\left(\int_{\mathbb{R}}(s-x)_{+}^{H / 2-1} f(x) d x\right)^{2} d s
\end{aligned}
$$

whence $a(s)=\int_{\mathbb{R}}(s-x)_{+}^{H / 2-1} f(x) d x=0$ for almost all $x \in[0,1]$ (in fact, for all $x \in[0,1]$, since $a$ is continuous as a linear combination of continuous functions). For $s \in[0,1 / 3]$, we have $a(s)=2\left(\alpha_{1}+\alpha_{2}\right) s^{H / 2} / H$, whence $\alpha_{1}+\alpha_{2}=0$. If $s \in(1 / 3,2 / 3)$, then (according to what we proved above) $a(s)=2 \alpha_{3}(s-1 / 3)^{H / 2} / H$, whence $\alpha_{3}=0$. Finally, $a(s)=2 \alpha_{1}(s-2 / 3)^{H / 2} / H$ for $s \in[2 / 3,1]$, whence $\alpha_{1}=-\alpha_{2}=0$. This contradiction proves Theorem 3.1 .

Theorem 3.2. A multifractional Rosenblatt process defined by equality (6) has a square integrable local time in each finite interval $[0, T]$.

Proof. As in the proof of the preceding theorem, we need to check condition (5) for $Z=X$ and $A=[0, T]$. In fact, it is sufficient to check it for $A=[a, a+\Delta]$, where $a \in[0, T-\Delta]$ is arbitrary and $\Delta>0$ is sufficiently small. The local time in the interval $[0, T]$ exists and is square integrable as a sum of local times $L([0, \Delta], x), L([\Delta, 2 \Delta], x), \ldots$, $L([(n-1) \Delta, n \Delta], x)$, and $L([n \Delta, T], x)$, where $n=[T / \Delta]$.

For $|t-s|<\Delta$ and $t>s$,

$$
\mathrm{E}\left[e^{i v\left(X_{t}-X_{s}\right)}\right]=\mathrm{E}\left[e^{i v I_{2}(g)}\right] \leq\left(1+64 v^{6} \lambda_{1, g}^{2} \lambda_{2, g}^{2} \lambda_{3, g}^{2}\right)^{-1 / 4},
$$

where $g_{t, s}(x, y)$ is the integral kernel corresponding to $X_{t}-X_{s}$. We represent the latter kernel as the sum $g_{1}(x, y)+g_{2}(x, y)$, where

$$
\begin{aligned}
& g_{1}(x, y)=\int_{s}^{t} f_{H(t)}(u, x, y) d u \\
& g_{2}(x, y)=\int_{0}^{s}\left(f_{H(t)}(u, x, y)-f_{H(s)}(u, x, y)\right) d u .
\end{aligned}
$$

It is clear that

$$
\left|\lambda_{k, g_{t, s}}\right| \geq\left|\lambda_{k, g_{1}}\right|-\left\|M_{g_{2}}|\geq| \lambda_{k, g_{1}} \mid-\right\| g_{2} \| .
$$

The bound $\left\|g_{2}\right\| \leq C|H(t)-H(s)| \leq C(t-s)^{\gamma}$ is established already in the proof of Proposition 2.3. On the other hand, $I_{2}\left(c(H(t)) g_{1}\right)=Z_{t}^{H(t)}-Z_{s}^{H(t)}$ has the same distribution as $(t-s)^{H(t)} Z_{1}^{H(t)}$. Thus

$$
\lambda_{k, g_{1}}=\frac{1}{c(H(t))}(t-s)^{H(t)} \lambda_{k, \phi_{H(t)}} \geq C(t-s)^{\check{H}} \lambda_{k, \phi_{H(t)}},
$$

where $\phi_{H(t)}$ is the kernel corresponding to $Z_{1}^{H(t)}$. The proof of Theorem 3.1 implies that $\lambda_{k, \phi_{H(t)}}>0, k=1,2,3$. Moreover $\inf _{t \in[0, T]} \lambda_{k, \phi_{H(t)}}>0, k=1,2,3$, since $\lambda_{k, f}$ continuously depends on $f$ (as above, we can write $\left|\lambda_{k, f_{1}}-\lambda_{k, f_{2}}\right| \leq\left\|f_{1}-f_{2}\right\|$ ) and since $\phi_{H(\cdot)}:[0, T] \rightarrow \widehat{L}^{2}\left(\mathbb{R}^{2}\right)$ is continuous (the continuity of $\phi_{H(\cdot)}$ follows from Proposition 2.3 and from the continuity of $H(t)$ and $c(H))$. 
Finally, $\lambda_{k, g_{1}} \geq C(t-s)^{\check{H}}$ for $k=1,2,3$. This together with

$$
\left\|g_{2}\right\| \leq C|H(t)-H(s)| \leq C(t-s)^{\gamma}=o\left((t-s)^{\check{H}}\right)
$$

and inequality (9) implies the bound $\left|\lambda_{k, g_{t, s}}\right| \geq C(t-s)^{\check{H}}, k=1,2,3$, for sufficiently small $\Delta$. Hence

$$
\mathrm{E}\left[e^{i v\left(X_{t}-X_{s}\right)}\right] \leq\left(1+C v^{6}(t-s)^{6 \check{H}}\right)^{-1 / 4}
$$

The rest of the proof is analogous to that of Theorem 3.1

\section{BIBLIOGRAPHY}

1. J. Albin, A note on Rosenblatt distributions, Stat. Probab. Lett. 40 (1998), no. 1, 83-91. MR 1650532 (2000b:60101)

2. S. M. Berman, Local times and sample function properties of stationary Gaussian processes, Trans. Amer. Math. Soc. 137 (1969), 277-299. MR0239652(39:1009)

3. R. Dobrushin and P. Major, Non-central limit theorems for non-linear functionals of Gaussian fields, Z. Wahrscheinlichkeitstheor. Verw. Geb. 50 (1979), 27-52. MR550122 (81i:60019)

4. A. Garsia and E. Rodemich, Monotonicity of certain functionals under rearrangement, Ann. Inst. Fourier 24 (1974), no. 2, 67-116. MR0414802 (54:2894)

5. Y. Mishura, Stochastic Calculus for Fractional Brownian Motion and Related Processes, Lecture Notes in Mathematics, vol. 1929, Springer, Berlin, 2008. MR2378138 (2008m:60064)

6. G. Peccati and M. S. Taqqu, Wiener Chaos: Moments, Cumulants and Diagrams. A Survey with Computer Implementation, Springer-Verlag, Italy, Milan-Dordrecht-Heidelberg-LondonNew York, 2011. MR2791919

7. V. Pipiras, Wavelet-type expansion of the Rosenblatt process, J. Fourier Anal. Appl. 10 (2004), no. 6, 599-634. MR2105535 (2005i:60076)

8. M. Rosenblatt, Independence and dependence, Proc. 4th Berkeley Symp. Math. Stat. Probab., vol. 2, 1961, pp. 431-443. MR0133863 (24:A3687)

9. G. Samorodnitsky and M. S. Taqqu, Stable non-Gaussian Random Processes: Stochastic Models with Infinite Variance, Stochastic Modeling, Chapman \& Hall, New York, 1994. MR1280932 (95f:60024)

10. M. S. Taqqu, Weak convergence to fractional Brownian motion and to the Rosenblatt process, Z. Wahrscheinlichkeitstheor. Verw. Geb. 31 (1975), 287-302. MR0400329 (53:4164)

11. M. S. Taqqu, Convergence of integrated processes of arbitrary Hermite rank, Z. Wahrscheinlichkeitstheor. Verw. Geb. 50 (1979), 53-83. MR550123 (81i:60020)

12. S. Torres and C. A. Tudor, Donsker type theorem for the Rosenblatt process and a binary market model, Stochastic Anal. Appl. 27 (2009), no. 3, 555-573. MR2523182(2010g:60075)

13. C. A. Tudor, Analysis of the Rosenblatt process, ESAIM, Probab. Stat. 12 (2008), 230-257. MR:2374640 (2008m:60067)

14. Svante Janson, Gaussian Hilbert Spaces, Cambridge Tracts in Mathematics, vol. 129, Cambridge University Press, Cambridge, 1997. MR.1474726 (99f:60082)

Department of Probability Theory, Statistics, and Actuarial Mathematics, Faculty for Mechanics and Mathematics, National Taras Shevchenko University, Academician Glushkov Avenue 2, Kiev 03127, Ukraine

E-mail address: zhora@univ.kiev.ua 